mmol/mol. Patients were followed up for major adverse cardiovascular events (MACE) including cardiovascular death, heart failure hospitalisation, non-fatal $\mathrm{MI}$ and non-fatal stroke. CMR studies were performed on a Siemens Prisma 3T scanner (Siemens Healthineers, Erlangen, Germany).

Results Of 343 patients, 176 were normoglycaemic and 167 dysglycaemic. During follow up (median 623 days) there were 35 MACE events in 30 patients, including 23 heart failure hospitalisations $(6.7 \%), 4$ strokes $(1.1 \%), 7$ cardiovascular deaths $(2.0 \%)$ and $1(0.3 \%)$ acute coronary syndrome. Univariate Cox regression analysis showed left ventricle ejection fraction (LVEF), right ventricle ejection fraction (RVEF), native T1, extracellular volume fraction, myocardial perfusion reserve (MPR) and the presence of occult IHD all to have significant association with MACE. However MPR was only associated with MACE in dysglycaemic patients (hazard ratio (HR) 0.19 , 95\% confidence interval (CI) $0.08-0.46, \mathrm{P}<0.001$ ) and occult IHD was only associated with MACE in normoglycaemic patients (HR 3.45, 95\% CI 1.23-9.71, $\mathrm{P}=0.02$ ) (figure 1). The relationship between MPR and MACE in dysglycaemic patients was still significant even after correction for LVEF, RVEF and HbA1c (HR 0.553, 95\% CI 0.318-0.962, $\mathrm{P}=0.036$ ).

Conclusions In patients with a recent diagnosis of heart failure, impairment of myocardial microvascular function is associated with adverse outcomes in dysglycaemic but not normoglycaemic patients, possibly explaining the excess risk in these patients. Further studies are needed to confirm these findings and establish if impaired microvascular function or associated outcomes can be altered by medical therapy.

\section{A MEDICAL DEVICE-GRADE T2 PHANTOM FOR QUALITY ASSURANCE OF INFLAMMATION IMAGING BY CMR}

${ }^{1}$ Massimiliano Fornasiero, ${ }^{2,3}$ lain Pierce, ${ }^{2-5}$ Matthew Webber, ${ }^{6}$ Kathryn E Keenan, ${ }^{6}$ Karl F Stupic, ${ }^{7}$ Rüdiger Bruehl, ${ }^{7}$ Bernd Ittermann, ${ }^{8}$ Wenjie Pang, ${ }^{3,5}$ Alun D Hughes, ${ }^{9}$ Reza Nezafat, ${ }^{10}$ Peter Kellman, ${ }^{2,3}$ James C Moon* ${ }^{3,4,5}$ Gabriella Captur*. ${ }^{1}$ UCL Medical School, 74 Huntley Street, London, WCIE 6DE, UK; ${ }^{2}$ Barts Heart Centre, Barts Health NHS Trust, West Smithfield, London, ECIA 7BE, UK; ${ }^{3}$ Institute of Cardiovascular Science, University College London, Huntley Street, London, WC1E 6DD, UK; ${ }^{4}$ Centre for Inherited Heart Muscle Conditions, Department of Cardiology, Royal Free London NHS Foundation Trust, Pond Street, London, NW3 2QG, UK; ${ }^{5}$ Medical Research Council Unit for Lifelong Health and Ageing at UCL, 1-19 Torrington Place, WC1E 7HB, London, UK; ${ }^{6}$ National Institute of Standards and Technology (NIST), 325 Broadway, Boulder, CO 80305, USA; 7Physikalisch-Technische Bundesanstalt (PTB), Abbestraße 2-12, D-10587 BerlinCharlottenburg, Germany; ${ }^{8}$ Resonance Health (RH), 141 Burswood Road, Burswood, WA 6100, Australia; ${ }^{9}$ Department of Medicine, Beth Israel Deaconess Medical Center, Harvard Medical School, 330 Brookline Ave, Boston, MA 02215, USA; ${ }^{10}$ National Heart, Lung and Blood Institute, National Institutes of Health (NIH), Rockville Pike, Bethesda, Maryland 20892, USA

\subsection{6/heartjnl-2021-BSCMR.11}

Introduction Cardiovascular magnetic resonance (CMR) T2 mapping is key to quantifying myocardial inflammation. Use of T2 mapping in clinical studies is burgeoning but in the absence of a quality control system, single-center findings are not generalizable and longitudinal studies cannot reliably track alterations in T2 times reflecting the inflammatory state of the myocardium.

Aim We used our expertise gained from the development of the T1 Mapping and Extracellular Volume (T1MES) phantom, to develop a dedicated T2 mapping CMR phantom to medical device standards.

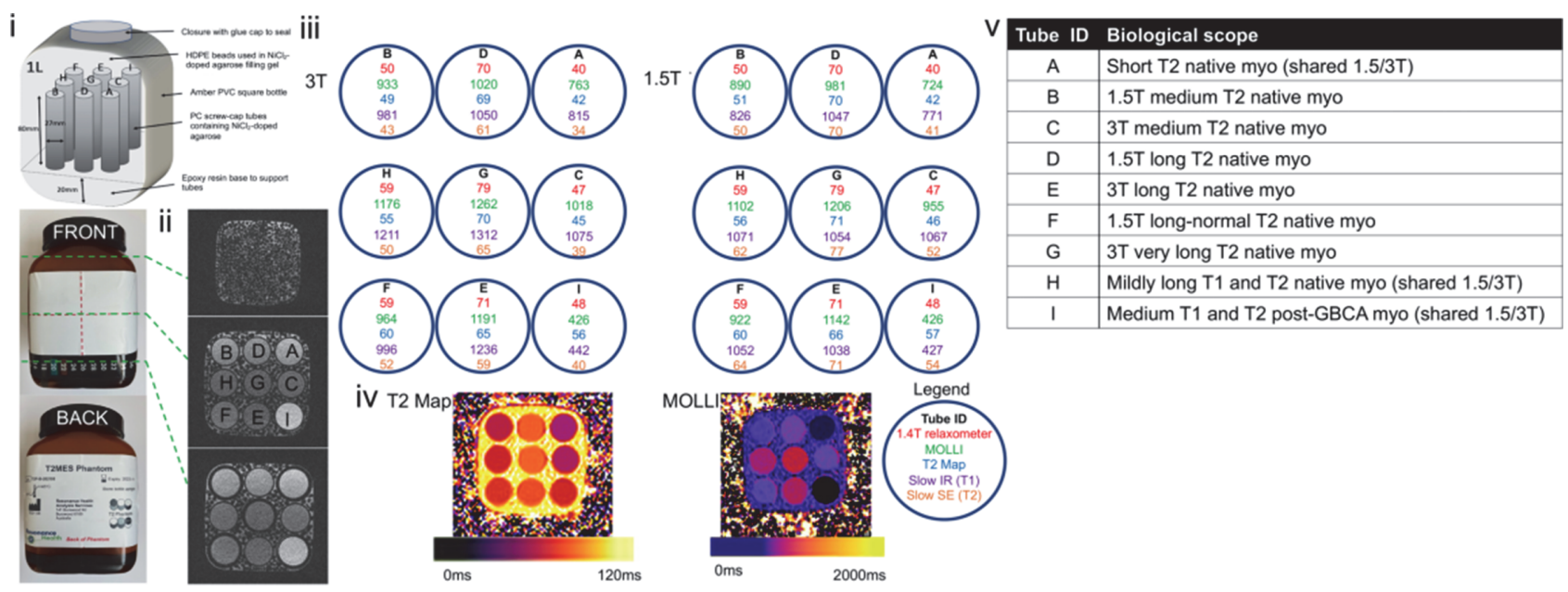

Abstract 11 Figure 1 (i) Schematic (not to scale) showing the internal and external phantom structure. (ii) Phantom front view showing isocentre line and liquid crystal display thermometer. (iii) $\mathrm{T} 1$ and $\mathrm{T} 2$ times in the T2 phantom as measured at 3T and 1.5T. Slow scan reference data for T1|T2 is displayed in purple|orange (for T1 by slow IRSE and for T2 by slow SE, RR interval $900 \mathrm{~ms}$ at $22^{\circ} \mathrm{C}$ ); $\mathrm{T} 1$ times shown in green were obtained using a 5(3)3 256-matrix RR $=900$ ms variant of MOLLI adapted for native T1 mapping; T2 times in blue are obtained using a T2 mapping sequence (SSFP); T2 times in red were obtained by the manufacturer in Australia using a $1.4 \mathrm{~T}$ Bruker minispec relaxometer at $22^{\circ} \mathrm{C}$. Tube arrangement is such that the more temperature-dependent and therefore unstable long-T1 tubes are away from the corners and towards the middle of the $3 \mathrm{X} 3$ array. (iv) Exemplar T2 and T1 maps on a Siemens 3T Prisma clinical CMR scanner. (v) The 9 relaxometry scopes per tube explained. FA = Flip angle; GBCA = Gadolinium-based contrast agent; HDPE=high-density polyethylene; IRSE = inversion recovery spin echo; MOLLI=modified Look-Locker inversion recovery; myo = myocardium; $\mathrm{PVC}=$ polyvinyl chloride; $\mathrm{PC}=$ polycarbonate; $\mathrm{RR}=$ inter-beat interval; $\mathrm{SE}=$ spin echo; SSFP=steady-state free precession; T=Tesla. 
Method A design collaboration including a specialist MRI small-medium enterprise, clinicians, physicists and national metrology institutes was formed. A T2 mapping phantom (figure 1 i) was designed to cover clinically relevant T1 and T2 times in native and post-contrast myocardium across fieldstrengths (figure 1 iii,v). Two earlier prototypes had been manufactured and tested, with the third and final one being reported here.

Results The T2 mapping phantom which can be used at both 1.5 and 3 Tesla is an agarose gel-based phantom using nickel chloride as the paramagnetic relaxation modifier. It contains nine differently-doped agarose gel tubes embedded in a gel/ beads matrix.

The phantom was free of air bubbles and susceptibility artifacts at both field strengths (figure 1 ii) and T2 maps were free from off-resonance artifacts (figure 1 iv). The incorporation of high-density polyethylene beads in the main gel fill was effective at flattening the $B_{0}$ and $B_{1}$ fields (figure $2 \mathrm{i}$,ii). T1 and T2 times measured in the phantom showed coefficients of variation of $\leq 1 \%$ between repeat scans indicating good short-term reproducibility. Temperature dependency experiments conducted at the national metrology institutes
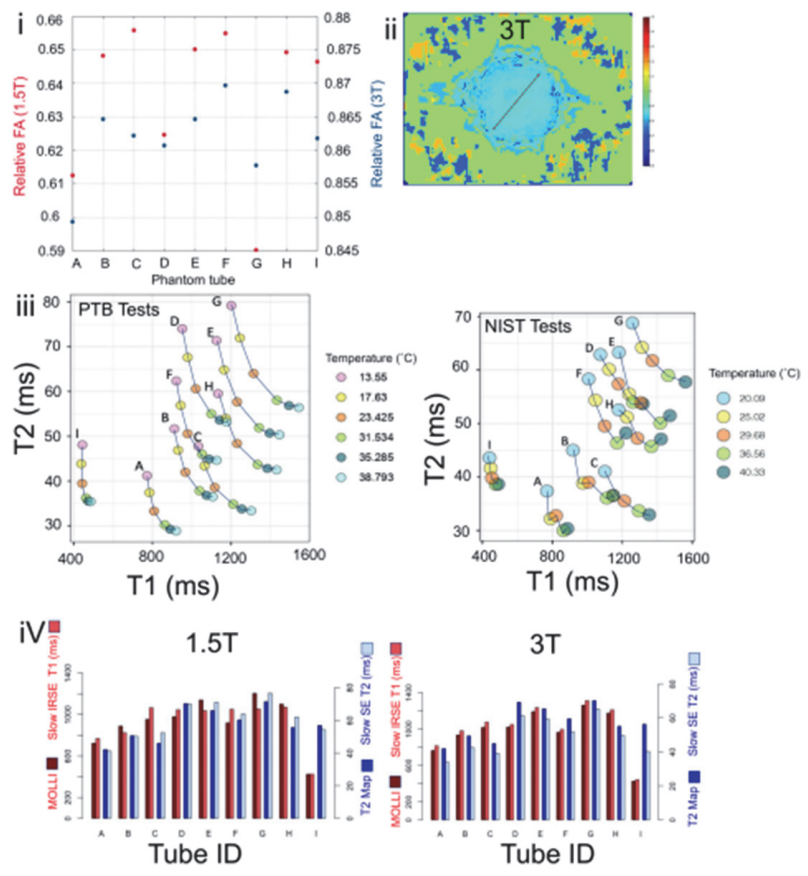

Abstract 11 Figure 2 (i) $B_{0}$ field homogeneity across the nine phantom compartments as a measure of off-resonance in hertz at $1.5 \mathrm{~T}$ (red) and $3 \mathrm{~T}$ (blue). These are extremely small shifts in frequency (e. g., $10 \mathrm{~Hz}=0.08 \mathrm{ppm}$ at $3 \mathrm{~T}$ ) and should not be regarded as significantly different between the tube compartments. (ii) T2 phantom field map at 3T showing $B_{1}$ homogeneity across the device cross-section. (iii) Temperature tests carried out at PTB-German Physikalisch-Technische Bundesanstalt (Left) -using a 3T Siemens Magnetom Verio system (VB17) and a 12-channel head coil and at NIST-US National Institute of Standards and Technology (Right) - using Agilent 3T small bore scanner. T1 was measured by IRSE (TR $[\mathrm{s}]=10, \mathrm{TI}[\mathrm{ms}]=50,75,100$, $125,150,250,500,1000,1500,2000,3000,6000)$ and T2 by SE (TR $[\mathrm{s}]=10, \mathrm{TE}[\mathrm{ms}]=14,28,56,112,224)$; resolution: $0.5 \times 0.5 \mathrm{~mm}$; slice thickness: $2 \mathrm{~mm}$. (iv) Comparison of reference T1|T2 times (by IRSE/SE respectively) to those obtained by MOLLI (5(3)3) and T2 mapping (SSFP) at 1.5T (Siemens Aera operating VE11C) and 3T (Siemens Prisma operating VE11C). TE=echo time; TR=repetition time. Other abbreviations as in figure 1. (figure 2 iii) confirmed that over the range $13-40^{\circ} \mathrm{C}$ the short-T1/2 tubes were more stable with temperature than the long-T1/2 tubes.

Conclusion The program has developed a T2 mapping phantom for CMR replicating clinically relevant T1/T2 times across myocardial health and disease. The device will be shortly listed under the Food and Drug Administration (FDA) database and Conformité Européene (CE) marking. Reproducible mass manufacture of this phantom may now commence to support the use of T2 mapping in longitudinal cohort studies, multicentre research or inflammation imaging.

\section{MYOCARDIAL INFLAMMATION AND DIFFUSE FIBROSIS UNDERPIN THE ELECTROPHYSIOLOGICAL DERANGEMENTS OF THE AGEING HUMAN HEART-A CMR-ECGI STUDY}

1,2,3,4 Matthew Webber, ${ }^{1,2}$ George Joy, ${ }^{3}$ Debbie Falconer, ${ }^{5}$ Xuyuan Tao, 1,2 lain Pierce, ${ }^{1,2}$ James C Moon, ${ }^{2,4}$ Alun D Hughes, ${ }^{1,2}$ Pier D Lambiase, ${ }^{6,7}$ Yoram Rudy* ${ }^{2,4}$ Michele Orini ${ }^{*}{ }^{2,3,4}$ Gabriella Captur. ${ }^{1}$ Barts Heart Centre, Barts Health NHS Trust, West Smithfield, ECIA 7BE, London, UK; ${ }^{2}$ Institute of Cardiovascular Science, University College London, Huntley Street, WC1E 6DD, London, UK; ${ }^{3}$ Centre for Inherited Heart Muscle Conditions, Department of Cardiology, Royal Free London NHS Foundation Trust, Pond Street, NW3 2QG, London, UK; ${ }^{4}$ Medical Research Council Unit for Lifelong Health and Ageing at UCL, 1-19 Torrington Place, WC1E 7HB, London, UK; ${ }^{5} E$ cole Nationale Supérieure des Arts et Industries Textiles, 2 Allée Louise et Victor Champier, 59056 Roubaix CEDEX 1, France; ${ }^{6}$ Cardiac Bioelectricity and Arrhythmia Center, Washington University, St Louis, MO 63130, USA; ${ }^{7}$ Department of Biomedical Engineering, Washington University, St. Louis, MO 63130, USA

10.1136/heartjnl-2021-BSCMR.12

Background Susceptibility to malignant ventricular arrhythmias can be predicted by activation recovery interval (ARI) and repolarisation time (RT) biomarkers noninvasively derived by electrocardiographic imaging (ECGI). The interaction between cardiac structural and electrophysiological changes during aging is unclear. We used our recently developed cardiovascular magnetic resonance (CMR)-ECGI vest to understand how the ageing myocardial substrate assessed by CMR interacts with these ECGI biomarkers under complete physiological conditions.

Methods CMR-ECGI at 3 Tesla was prospectively performed (figure 1). 256-Lead body surface potentials were recorded and co-registered with CMR-derived heart-torso geometries. Epicardial unipolar potentials were reconstructed using stateof-the-art ECGI algorithms to derive whole-heart electrophysiological maps (figure 2).

Results 45 Participants were recruited: 29 older persons from a population-based cohort (all 75 years; $55 \%$ male) and 16 young healthy volunteers ( $34 \pm 3$ years, 62\% male). Compared to young participants, older ones showed longer T1 $(1300 \pm 38.4 \mathrm{~ms}$ vs $1280 \pm 46.5 \mathrm{~ms}, p=0.020)$ and $\mathrm{T}_{2}$ $(46.1 \pm 0.06 \mathrm{~ms}$ vs $42.9 \pm 2.3 \mathrm{~ms}, p=0.001)$ and higher extracellular volume (ECV, 26.6 $\pm 1.9 \%$ vs $22.6 \pm 3.5 \%$, $p<0.001)$. Activation time (AT) was prolonged in older compared to younger hearts $(41.8 \pm 19.7 \mathrm{~ms}$ vs $36.8 \pm 20.0$ $\mathrm{ms}, p=0.042$ ) with similar but weaker trends observed for RT and ARI $(p=0.086, p=0.160)$. Apart from left ventricular ejection fraction (LVEF, e.g. for ARI $\beta=1.9 \mathrm{~ms}[0.01-$ 3.7] $p=0.049$ ), no other standard CMR size/function parameter or binary presence of late gadolinium enhancement, showed an association with ECGI parameters. By contrast, native $\mathrm{T}_{1}, \mathrm{~T}_{2}$ and $\mathrm{ECV}$ were all associated with $\mathrm{RT}$ 